\title{
GRINDING SYNTHESIS OF 2-AMINO-4H-CHROMENES USING 3,3-(BUTANE-1,4-DIYL) BIS (1,2-DIMETHYL- $1 H$-IMIDAZOLE-3-IUM)Br-CAN AS A NOVEL REAGENT
}

\author{
MOHAMMAD NIKPASSAND ${ }^{*}$, , LEILA ZARE FEKRI ${ }^{b}$, PARVIN AHMADI \\ a'Department of Chemistry, Rasht Branch, Islamic Azad University, Rasht, Iran \\ ${ }^{b}$ Department of Chemistry, Payame Noor University, PO Box 19395-3697 Tehran, Iran
}

\begin{abstract}
A clean and environmentally benign route to 2 -amino- $4 H$-chromenes has been developed via three-component condensation reaction of various benzyl alcohols, malononitrile and 1-naphthol, using a catalytical amount of CAN and a reusable ionic liquid 3,3-(Butane-1,4-diyl)bis(1,2-dimethyl-1 H-imidazole-3-ium) bromide $([\mathrm{BDBDMIm}] \mathrm{Br})$ as a catalyst at room temperature. The present methodology offers several advantages such as solvent-free conditions, excellent yields, simple procedure, mild conditions and reduced environmental consequences. The ionic liquid was recovered and reused. All of synthesized compounds were characterized by IR, NMR and elemental analyses.
\end{abstract}

Keywords: Chromenes, Benzyl alcohols, Ionic Liquid, Multicomponent reaction, 1-Naphthol, Malononitrile.

\section{INTRODUCTION}

Chromene and its derivatives belong to a major class of natural heterocyclic compounds, which they occur widely in edible vegetables and fruits ${ }^{1,2}$. They frequently expose a variety of biological and pharmacological activities ${ }^{3}$. Based on the extensive researches, it has been observed that chromene derivatives include biological, such as antioxidant, spamolytic, anti-HIV, anticancer, antianaphylactic, antibacterial activity antihypertensive, anti-tubulin, antiviral, activator of potassium channels and inhibition of phosphodiesterase IV or dihydrofolate reductase, etc. ${ }^{4-12}$. As a result, a number of methodologies have been developed to synthesize chromene compounds ${ }^{13}$.

Several methods have been reported for the synthesis of 2-amino- $4 H$ chromene derivatives using malononitrile, resorcinol and aldehyde. Various catalysts such as $\mathrm{TFE}{ }^{14}, \mathrm{MgAl} / \mathrm{HT}{ }^{15}$, cetyltrimethylammonium bromide (CTABr) ${ }^{16}$ and tungstic acid functionalized mesoporous SBA-15 ${ }^{17}$ have been used for these reactions. Most of these reported methods require a long reaction time, high temperature, and unsatisfactory yields.

The use of ionic liquids as reaction media and catalyst can offer a solution to solvent emission and catalyst recycling problems. Ionic liquids are accompanied with some pluses or let us call them advantages; some of which are negligible vapor pressure, non-flammability, non- miscibility with non-polar solvents, reasonable thermal and chemical stability and recyclability 18, 19. They dissolve many organic and inorganic substrates and are tunable to specific chemical tasks ${ }^{20}$.

\section{EXPERIMENTAL}

\section{Materials and measurements}

Chemicals were purchased from Merck and Fluka. All solvents used were dried and distilled according to standard procedures. Thin Layer Chromatography (TLC) was done with TLC Silica gel 60; Aluminum sheet from Merck. Melting points were measured on an Electrothermal 9100 apparatus. IR spectra were determined on a Shimadzu FT-IR 8600 spectrophotometer. ${ }^{1} \mathrm{H}$ and ${ }^{13} \mathrm{C}$ NMR spectra were determined on a Bruker 400 DRX Avance instrument at 500 and $125 \mathrm{MHz}$. Elemental analyses were done on a CarloErba EA1110CNNO-S analyzer and agreed with the calculated values.

General procedure for the synthesis of 2-amino-4H-chromene 4a-p

A mixture containing benzyl alcohol $(2.0 \mathrm{mmol}), 1$-naphthol $(2.2 \mathrm{mmol})$, malononitrile $(2.0 \mathrm{mmol})$ and $4 \mathrm{mmol} \%$ of [BDBDMIm] $\mathrm{Br}^{21}$ and $0.05 \mathrm{~g}$ of CAN was stirred at room temperature for the required reaction times. The progress of the reaction was monitored by TLC (EtOAc: petroleum ether 1:2). After completion of the reaction, we extracted the product with $\mathrm{CHCl}_{3} / \mathrm{H}_{2} \mathrm{O}$. After separation of phases and evaporation of the organic phase and recrystallization of the residue, the pure product was obtained. The aqueous phase was concentrated under reduced pressure, washed with $\mathrm{Et}_{2} \mathrm{O}$, and evaporated under reduced pressure to recover the ionic liquid for subsequent use.

Selected data:

2-Amino-3-cyano-4-(4-nitrophenyl)-4H-benzo[ $h]$ chromene(4a):

Yellow solid, $\mathrm{mp} 239-241^{\circ} \mathrm{C}$; FT-IR (KBr): v 3350, 3550, 2200, 1600, $1670,1510,1350 \mathrm{~cm}^{-1} .{ }^{1} \mathrm{H}$ NMR $\left(400 \mathrm{MHz}, \mathrm{CDCl}_{3}\right): \delta=4.87\left(\mathrm{~s}, 2 \mathrm{H}, \mathrm{NH}_{2}\right)$, $5.12(\mathrm{~s}, 1 \mathrm{H}), 6.83(\mathrm{~d}, 1 \mathrm{H}, J=8.2 \mathrm{~Hz}),, 7.42(\mathrm{~d}, 1 \mathrm{H}, J=8.4 \mathrm{~Hz}), 7.54-7.67(\mathrm{~m}$, $3 \mathrm{H}), 7.87(\mathrm{~d}, 1 \mathrm{H}, J=7.8 \mathrm{~Hz}), 8.19$ (d, $2 \mathrm{H}, J=8.4 \mathrm{~Hz}), 8.22(\mathrm{~d}, 1 \mathrm{H}, J=8.2 \mathrm{~Hz})$ ppm. ${ }^{13} \mathrm{C} \mathrm{NMR}\left(\mathrm{CDCl}_{3}, 100 \mathrm{MHz}\right): \delta=41.3,61.6,117.4,119.8,121.6,123.8$, 125.4, 125.6, 125.8, 127.3, 128.1, 128.7, 129.2, 130.6, 133.7, 147.8, 151.9, 159.3 ppm. Anal Calc. for $\mathrm{C}_{20} \mathrm{H}_{13} \mathrm{~N}_{3} \mathrm{O}_{3}: \mathrm{C}, 69.96 ; \mathrm{H}, 3.82 ; \mathrm{N}, 12.24$. Found: $\mathrm{C}$, $70.09 ; \mathrm{H}, 3.92 ; \mathrm{N}, 12.37$.

2-Amino-3-cyano-4-(4-chlorphenyl)-4H-benzo $[h]$ chromene (4b)

Yellow solid, mp $231-233^{\circ} \mathrm{C}$; FT-IR (KBr): v 3320, 3420, 2200, 1670, $1600,1570 \mathrm{~cm}^{-1} .{ }^{1} \mathrm{H}$ NMR $\left(\mathrm{CDCl}_{3} 400 \mathrm{MHz}\right): \delta=4.79\left(\mathrm{~s}, 2 \mathrm{H}, \mathrm{NH}_{2}\right), 4.96(\mathrm{~s}$, $1 \mathrm{H}), 7.09(\mathrm{~d}, 1 \mathrm{H}, J=8.4 \mathrm{~Hz}), 7.27$ (d, $2 \mathrm{H}, J=8.4 \mathrm{~Hz}), 7.38(\mathrm{~d}, 2 \mathrm{H}, J=8.4$ $\mathrm{Hz}), 7.57-7.66(\mathrm{~m}, 3 \mathrm{H}), 7.89(\mathrm{~d}, 1 \mathrm{H}, J=8.0 \mathrm{~Hz}), 8.23(\mathrm{~d}, 1 \mathrm{H}, J=8.0 \mathrm{~Hz})$ $\mathrm{ppm} ;{ }^{13} \mathrm{C}$ NMR $\left(\mathrm{CDC}_{13}, 100 \mathrm{MHz}\right): \delta=41.2,61.2,117.5,119.2,120.6,122.6$, $123.3,125.2,127.4,128.4,128.9,129.2,130.2,132.8,133.4,141.5,142.7$, 158.6 ppm. Anal Calc. for $\mathrm{C}_{20} \mathrm{H}_{13} \mathrm{ClN}_{2} \mathrm{O}: \mathrm{C}, 72.18 ; \mathrm{H}, 3.94 ; \mathrm{N}, 8.42$. Found: $\mathrm{C}$, $72.31 ; \mathrm{H}, 3.76 ; \mathrm{N}, 8.53$. (4c)

2-Amino-4-(3-hydroxyphenyl)-4H-benzo $[h]$ chromene-3-carbonitrile

Yellow solid, mp $252-254^{\circ} \mathrm{C}$; FT-IR (KBr): v 3370, 3420, 3320, 2200, $1580,1650 \mathrm{~cm}^{-1} .{ }^{1} \mathrm{H}$ NMR $\left(\mathrm{CDCl}_{3}, 400 \mathrm{MHz},\right): \delta=4.77(\mathrm{~s}, 1 \mathrm{H}), 6.62-6.67(\mathrm{~m}$, $2 \mathrm{H}), 6.76(\mathrm{~d}, 1 \mathrm{H}, J=7.6 \mathrm{~Hz}), 7.03-7.18(\mathrm{~m}, 2 \mathrm{H}), 7.17\left(\mathrm{~s}, 2 \mathrm{H}, \mathrm{NH}_{2}\right), 7.58-7.68$ $(\mathrm{m}, 3 \mathrm{H}), 7.84(\mathrm{~d}, 1 \mathrm{H}, J=8.0 \mathrm{~Hz}), 8.25(\mathrm{~d}, 1 \mathrm{H}, J=8.4 \mathrm{~Hz}), 9.39(\mathrm{~s}, 1 \mathrm{H}, \mathrm{OH})$ ppm. 13C NMR $\left(\mathrm{CDCl}_{3}, 100 \mathrm{MHz}\right): \delta=48.6,61.7,116.9,119.2,121.8,123.3$, $123.5,123.7,125.1,125.2,127.1,127.3,129.5,131.5,133.3,134.1,145.7$, 146.5, 148.9, 160.2 ppm. Anal Calc. for $\mathrm{C}_{20} \mathrm{H}_{14} \mathrm{~N}_{2} \mathrm{O}_{2}: \mathrm{C}, 76.42 ; \mathrm{H}, 4.49 ; \mathrm{N}, 8.91$. Found: C, 76.49; H, 4.56; N, 8.76.

2-Amino-3-cyano-4-(4-methylphenyl)-4H-benzo[ $h]$ chromene (4d)

Yellow solid, $\mathrm{mp} 206-208^{\circ} \mathrm{C}$; FT-IR (KBr): $v 3435,3320,2180,1657 \mathrm{~cm}^{-1}$. ${ }^{1} \mathrm{H} \mathrm{NMR}\left(\mathrm{CDCl}_{3}, 400 \mathrm{MHz}\right): \delta=2.32(\mathrm{~s}, 3 \mathrm{H}, \mathrm{CH} 3), 4.73\left(\mathrm{~s}, 2 \mathrm{H}, \mathrm{NH}_{2}\right), 4.84(\mathrm{~s}$ $1 \mathrm{H}), 7.05(\mathrm{~d}, 1 \mathrm{H}, J=8.2 \mathrm{~Hz}), 7.13-7.45(\mathrm{~m}, 4 \mathrm{H}), 7.47-7.66(\mathrm{~m}, 3 \mathrm{H}), 7.74(\mathrm{~d}$, $1 \mathrm{H}, J=8.2 \mathrm{~Hz}), 8.23(\mathrm{~d}, 1 \mathrm{H}, J=8.4 \mathrm{~Hz}) \mathrm{ppm} .{ }^{13} \mathrm{C}$ NMR $(100 \mathrm{MHz}, \mathrm{CDCl} 3)$ : $\delta=22.9\left(\mathrm{CH}_{3}\right), 42.6,61.8,116.6,119.0,120.8,122.9,124.4,126.6,126.8$, $127.2,128.0,128.5,128.9,130.4,133.6,137.7,142.5,159.6$ ppm. Anal Calc. for $\mathrm{C}_{21} \mathrm{H}_{16} \mathrm{~N}_{2} \mathrm{O}: \mathrm{C}, 80.75 ; \mathrm{H}, 5.16 ; \mathrm{N}, 8.97$. Found: $\mathrm{C}, 80.61 ; \mathrm{H}, 5.24 ; \mathrm{N}, 9.07$.

2-Amino-3-cyano-4-(3-nitrophenyl)-4H-benzo[ $h]$ chromene (4e)

Yellow solid, $\mathrm{mp} 211-213^{\circ} \mathrm{C}$; FT-IR (KBr): v 3320, 3450, 2210, 1660, $1600,1520,1350 \mathrm{~cm}^{-1} ;{ }^{1} \mathrm{H}$ NMR $\left(\mathrm{CDCl}_{3}, 400 \mathrm{MHz}\right): \delta=4.93\left(\mathrm{~s}, 2 \mathrm{H}, \mathrm{NH}_{2}\right)$, $5.12(\mathrm{~s}, 1 \mathrm{H}, \mathrm{s}), 6.83(\mathrm{~d}, 1 \mathrm{H}, J=8.4 \mathrm{~Hz}), 7.51-7.63(\mathrm{~m}, 5 \mathrm{H}), 7.82(\mathrm{~d}, 1 \mathrm{H}, J=$ $7.6 \mathrm{~Hz}), 8.20(\mathrm{~m}, 2 \mathrm{H}), 8.25(\mathrm{~d}, 1 \mathrm{H}, J=7.8 \mathrm{~Hz}) \mathrm{ppm} .{ }^{13} \mathrm{C} \mathrm{NMR}(\mathrm{CDCl}, 100$ MHz): $\delta=41.4,61.3,116.6,119.6,121.4,123.3,123.7,123.8,125.3,125.6$, 127.3, 127.6, 129.6, 130.3, 133.7, 134.5, 145.3, 147.5, 149.2, 159.7 ppm. Anal Calc. for $\mathrm{C}_{20} \mathrm{H}_{13} \mathrm{~N}_{3} \mathrm{O}_{3}$ : C, 69.96; H, 3.82; N, 12.24. Found: C, 70.02; H, 3.93; $\mathrm{N}, 12.35$.

2-amino-4-(2-nitrophenyl)-4H-benzo[ $h]$ chromene-3-carbonitrile (4f)

Dark yellow solid, mp $234-236^{\circ} \mathrm{C}$; FT-IR (KBr): $v 3346,2202,1666,1525$ $\mathrm{cm}^{-1} ;{ }^{1} \mathrm{H}$ NMR $\left(\mathrm{CDCl}_{3}, 400 \mathrm{MHz}\right): \delta=1.55\left(\mathrm{~s}, 2 \mathrm{H}, \mathrm{NH}_{2}\right), 4.87(\mathrm{~s}, 1 \mathrm{H}), 7.13(\mathrm{~d}$, $1 \mathrm{H}, J=8.4 \mathrm{~Hz}), 7.33-7.87(\mathrm{~m}, 7 \mathrm{H}), 7.85(\mathrm{~d}, 1 \mathrm{H}, J=8.4 \mathrm{~Hz}), 8.19(\mathrm{~d}, 1 \mathrm{H}, J=$ $8.4 \mathrm{~Hz}) \mathrm{ppm} .{ }^{13} \mathrm{C}$ NMR $\left(\mathrm{CDCl}_{3}, 100 \mathrm{MHz}\right): \delta=46.8,61.6,117.8,119.2,121.1$, $123.4,123.7,123.8,125.3,125.6,127.7,127.8,129.2,130.4,133.3,134.8$, 145.7, 147.0, 149.2, 159.9 ppm. Anal Calc. for $\mathrm{C}_{20} \mathrm{H}_{13} \mathrm{~N}_{3} \mathrm{O}_{3}: \mathrm{C}, 69.96 ; \mathrm{H}, 3.82$; N, 12.24. Found: C, 69.85; H, 3.94; N, 12.18 . 

(4g)

2-amino-4-(2-chlorophenyl)-4H-benzo $[h]$ chromene-3-carbonitrile

White solid, $\mathrm{mp} 234-236^{\circ} \mathrm{C}$; FT-IR (KBr): v 3327, 2198, 1662, 1103, 750 $\mathrm{cm}^{-1} .{ }^{1} \mathrm{H}$ NMR $\left(\mathrm{CDCl}_{3}, 400 \mathrm{MHz}\right): \delta=1.59\left(\mathrm{~s}, 2 \mathrm{H}, \mathrm{NH}_{2}\right), 4.75(\mathrm{~s}, 1 \mathrm{H}), 7.15(\mathrm{~d}$, $1 \mathrm{H}, J=8.6 \mathrm{~Hz}$,), $7.23-7.65$ (m, $7 \mathrm{H}), 7.74$ (d, $1 \mathrm{H}, J=7.8 \mathrm{~Hz}), 8.17$ (d, $1 \mathrm{H}, J=$ 8.4Hz) ppm. ${ }^{13} \mathrm{C}$ NMR $\left(\mathrm{CDCl}_{3}, 100 \mathrm{MHz}\right): \delta=46.2,62.3,117.3,119.4,121.5$, $123.2,123.3,123.5,125.1,125.5,127.3,127.7,128.8,130.2,133.5,134.9$ 145.3, 147.7, 149.8, $159.1 \mathrm{ppm}$. Anal Calc. for $\mathrm{C}_{20} \mathrm{H}_{13} \mathrm{ClN}_{2} \mathrm{O}: \mathrm{C}, 72.18 ; \mathrm{H}, 3.94$; N, 8.42. Found: C, 72.31; H, 3.84; N, 8.59.

2-Amino-3-cyano-4-phenyl-4H-benzo $[h]$ chromene $(4 \mathrm{~h})$

Yellow solid, $\mathrm{mp} 209-211^{\circ} \mathrm{C}$; FT-IR $(\mathrm{KBr}): v$ 3310, 3430, 2190, 1550, $1532 \mathrm{~cm}^{-1} .{ }^{1} \mathrm{H}$ NMR $\left(\mathrm{CDCl}_{3}, 400 \mathrm{MHz}\right): \delta=4.72\left(\mathrm{~s}, 2 \mathrm{H}, \mathrm{NH}_{2}\right), 4.82(\mathrm{~s}, 1 \mathrm{H})$, $7.07(\mathrm{~d}, 1 \mathrm{H}, J=8.4 \mathrm{~Hz}), 7.12-7.35(\mathrm{~m}, 5 \mathrm{H}), 7.39-7.67(\mathrm{~m}, 3 \mathrm{H}), 7.76(\mathrm{~d}, 1 \mathrm{H}$, $J=8.2 \mathrm{~Hz}), 8.23(\mathrm{~d}, 1 \mathrm{H}, J=7.8 \mathrm{~Hz}) \mathrm{ppm} .{ }^{13} \mathrm{C} \mathrm{NMR}\left(\mathrm{CDCl}_{3}, 100 \mathrm{MHz}\right): \delta=$ $42.9,61.5,117.8,119.4,120.7,123.4,124.5,126.3,126.4,126.8,127.2,127.4$ $128.5,129.4,133.6,142.4,144.3,159.3 \mathrm{ppm}$. Anal Calc. for $\mathrm{C}_{20} \mathrm{H}_{14} \mathrm{~N}_{2} \mathrm{O}: \mathrm{C}$, 80.52; H, 4.73; N, 9.39. Found: C, 80.65; H, 4.86; N, 9.18.

2-amino-4-(4-bromophenyl)-4H-benzo $[h]$ chromene-3-carbonitrile (4i)

Dark yellow solid, mp 195-197 C; FT-IR (KBr): v 3350, 2191, 1651, 1101 $\mathrm{cm}^{-1} .{ }^{1} \mathrm{H}$ NMR $\left(\mathrm{CDCl}_{3}, 400 \mathrm{MHz}\right): \delta=1.56\left(\mathrm{~s}, 2 \mathrm{H}, \mathrm{NH}_{2}\right), 5.04(\mathrm{~s}, 1 \mathrm{H}), 6.95(\mathrm{~d}$, $1 \mathrm{H}, J=8.6 \mathrm{~Hz}), 7.63-6.96(\mathrm{~m}, 7 \mathrm{H}), 7.83(\mathrm{~d}, 1 \mathrm{H}, J=8.5 \mathrm{~Hz}), 8.23(\mathrm{~d}, 1 \mathrm{H}, J=$ $8.5 \mathrm{~Hz}) \mathrm{ppm} .{ }^{13} \mathrm{C}$ NMR $\left(\mathrm{CDCl}_{3}, 100 \mathrm{MHz}\right): \delta=51.2,61.9,113.0,118.8,121.9$, $129.4,123.9,125.4,125.5,125.8,127.6,128.3,128.6,130.9,133.6,146.7$, 152.3, 159.9 ppm. Anal Calc. for $\mathrm{C}_{20} \mathrm{H}_{13} \mathrm{BrN}_{2} \mathrm{O}$ : C, 63.68; H, 3.47; N, 7.43. Found: $\mathrm{C}, 63.82 ; \mathrm{H}, 3.32 ; \mathrm{N}, 7.53$.

2-amino-4-(4-methoxyphenyl)-4H-benzo[ $h]$ chromene-3-carbonitrile $(4 \mathrm{j})$

Yellow solid, mp $182-184^{\circ} \mathrm{C}$; FT-IR (KBr): v 3416, 3316, 2183, $1633 \mathrm{~cm}^{-1}$ ${ }^{1} \mathrm{H} \mathrm{NMR}\left(\mathrm{CDCl}_{3}, 400 \mathrm{MHz}\right): \delta=3.52\left(\mathrm{~s}, 3 \mathrm{H}, \mathrm{OCH}_{3}\right), 4.89\left(\mathrm{~s}, 2 \mathrm{H}, \mathrm{NH}_{2}\right), 4.83$ $(\mathrm{s}, 1 \mathrm{H}), 7.16(\mathrm{~d}, 1 \mathrm{H}, J=8.4 \mathrm{~Hz}), 7.25-7.36(\mathrm{~m}, 4 \mathrm{H}), 7.47-7.56(\mathrm{~m}, 3 \mathrm{H}), 7.75(\mathrm{~d}$, $1 \mathrm{H}, J=8.4 \mathrm{~Hz}), 8.24(\mathrm{~d}, 1 \mathrm{H}, J=8.2 \mathrm{~Hz}) \mathrm{ppm} .{ }^{13} \mathrm{C} \mathrm{NMR}\left(\mathrm{CDCl}_{3}, 100 \mathrm{MHz}\right)$ : $\delta=41.8,55.9\left(\mathrm{OCH}_{3}\right), 61.8,117.9,120.3,121.4,123.5,124.5,126.5,126.9$, $127.7,127.9,128.3,128.7,129.2,133.4,144.6,154.4,159.8$ ppm. Anal Calc. for $\mathrm{C}_{21} \mathrm{H}_{16} \mathrm{~N}_{2} \mathrm{O}_{2}$ : C, 76.81; H, 4.91; N, 8.53. Found: C, 76.86; H, 4.97; N, 8.71.

2-amino-4-(2-methoxyphenyl)-4H-benzo[ $h]$ chromene-3-carbonitrile (4k)

Yellow solid, mp $172-174^{\circ} \mathrm{C}$; FT-IR (KBr); v 3320, 3470, 2170, 1600, $1660 \mathrm{~cm}^{-1} .{ }^{1} \mathrm{H} N M R\left(\mathrm{CDCl}_{3}, 400 \mathrm{MHz}\right): \delta=3.81\left(\mathrm{~s}, 3 \mathrm{H}, \mathrm{OCH}_{3}\right), 5.26(\mathrm{~s}, 1 \mathrm{H})$ $6.88(\mathrm{~d}, 1 \mathrm{H}, J=6.8 \mathrm{~Hz}), 7.04(\mathrm{t}, 1 \mathrm{H}, J=7.6 \mathrm{~Hz}), 7.10\left(\mathrm{~s}, 2 \mathrm{H}, \mathrm{NH}_{2}\right), 7.12(\mathrm{~d}$, $1 \mathrm{H}, J=7.2 \mathrm{~Hz}), 7.13(\mathrm{~d}, 1 \mathrm{H}, J=8.1 \mathrm{~Hz}), 7.21(\mathrm{~d}, 1 \mathrm{H}, J=7.2 \mathrm{~Hz}), 7.56(\mathrm{t}, 2 \mathrm{H}$, $J=7.6 \mathrm{~Hz}), 7.63(\mathrm{t}, 1 \mathrm{H}, J=6.8 \mathrm{~Hz}), 7.86(\mathrm{~d}, 1 \mathrm{H}, J=8.0 \mathrm{~Hz}), 8.22(\mathrm{~d}, 1 \mathrm{H}, J=$ $8.0 \mathrm{~Hz}) \mathrm{ppm} .{ }^{13} \mathrm{C}$ NMR $\left(\mathrm{CDCl}_{3}, 100 \mathrm{MHz}\right): \delta=46.2,56.4\left(\mathrm{OCH}_{3}\right), 61.8,117.2$, $119.3,121.5,123.4,123.5,123.6,125.3,125.5,127.7,127.9,129.6,130.8$, 133.4, 134.7, 145.2, 147.6, 149.6, 159.2 ppm. Anal Calc. for $\mathrm{C}_{21} \mathrm{H}_{16} \mathrm{~N} 2 \mathrm{O} 2$ : C, $76.81 ; \mathrm{H}, 4.91 ; \mathrm{N}, 8.53$. Found: C, 76.96; H, 4.84; N, 8.62.

\section{RESULTS AND DISCUSSION}

Furthering the ongoing studies to synthesize heterocyclic and pharmaceutical compounds by mild and practical protocols ${ }^{21-24}$, the researchers would like to report the experimental results on the synthesis of 2-amino- $4 H$ chromenes, using various benzyl alcohols, 1-naphthol and malononitrile in the presence of bis ionic liquid 3,3-(Butane-1,4-diyl)bis(1,2-dimethyl-1Himidazole-3-ium) bromide ([BDBDMIm]Br) and CAN at room temperature (Scheme 1).

To check the effect of catalyst, the model reaction between 4-nitro benzyl alcohol, 1-naphthol and malononitrile in the presence of different acidic catalysts was carried out. All the reactions were carried out with catalytic amounts of catalysts. As shown in Table 1 the results gained with $0.04 \mathrm{mmol}$ of [BDBDMIm]Br (Table 1; Entry 11) were to a great extent satisfactory.

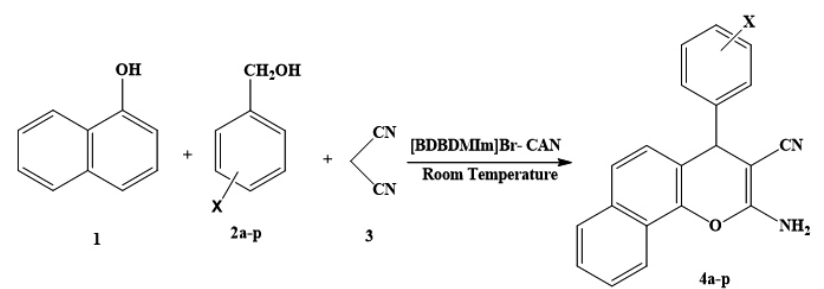
AN
Table 1 showed other interesting points that the ability and efficiency of catalyst $[\mathrm{BMIm}] \mathrm{Br}$, $[\mathrm{BMIm}] \mathrm{OH}$ and $[\mathrm{BMIm}] \mathrm{HSO}_{4}$ are somehow similar, while ionic liquid [BDBDMIm] Br was more efficient for the synthesis of 2-amino$4 H$-chromenes. The ionic liquid [BDBDMIm] $\mathrm{Br}$, rather than ionic liquids $[\mathrm{BMIm}] \mathrm{Br},[\mathrm{BMIm}] \mathrm{OH}$, and $[\mathrm{BMIm}] \mathrm{HSO}_{4}$, can accelerate the reaction time.

Table 1. Effect of catalyst on the synthesis of $\mathbf{4 a}$

\begin{tabular}{|c|c|c|c|c|c|}
\hline Entry & Catalyst & $\begin{array}{c}\text { Catalyst } \\
\text { amount } / 1 \text { mmol } \\
\text { of aldehyde }\end{array}$ & $\begin{array}{l}\text { Reaction } \\
\text { condition }\end{array}$ & $\begin{array}{l}\text { Time } \\
\text { (hr) }\end{array}$ & $\begin{array}{l}\text { Yield } \\
(\%)\end{array}$ \\
\hline 1 & $\mathrm{HCl}$ & 4drops & reflux & 12 & 52 \\
\hline 2 & $\mathrm{SiO}_{2}$ & $0.2 \mathrm{mmol}$ & reflux & 6 & 65 \\
\hline 3 & Montmorillonite K10 & $0.2 \mathrm{~g}$ & reflux & 4 & 71 \\
\hline 4 & Montmorillonite K10 & $0.2 \mathrm{~g}$ & reflux & 3.5 & 68 \\
\hline 5 & $\mathrm{Fe}_{3} \mathrm{O}_{4}$ & $0.2 \mathrm{mmol}$ & reflux & 7 & 48 \\
\hline 6 & $\mathrm{ZnCl}_{2}$ & $0.2 \mathrm{mmol}$ & reflux & 6 & 63 \\
\hline 7 & {$[\mathrm{BMIm}] \mathrm{Br}$} & $0.04 \mathrm{mmol}$ & neat, r.t. & 3 & 80 \\
\hline 8 & {$[\mathrm{BMIm}] \mathrm{OH}$} & $0.04 \mathrm{mmol}$ & neat, r.t. & 3 & 79 \\
\hline 9 & {$[\mathrm{BMIm}] \mathrm{HSO}_{4}$} & $0.04 \mathrm{mmol}$ & neat, r.t. & 3 & 82 \\
\hline 10 & [BDBDIm]Br & $0.02 \mathrm{mmol}$ & neat, r.t. & 1.5 & 87 \\
\hline 11 & [BDBDIm]Br & $0.04 \mathrm{mmol}$ & neat, r.t. & 1 & 96 \\
\hline 12 & {$[\mathrm{BDBDIm}] \mathrm{Br}$} & $0.06 \mathrm{mmol}$ & neat, r.t. & 1 & 96 \\
\hline
\end{tabular}

a. solvent in the entries 1- 6 was water ${ }^{\text {b. }} 0.05 \mathrm{~g}$ of CAN was used in the all of reactions

To investigate the efficiency and generality of the reaction, various benzyl alcohols were combined with 1-naphthol and malononitrile in the presence of [BDBDMIm] Br-CAN at room temperature. The results are summarized in Table 2. As indicated in Table 2, the electron withdrawing substituents can facilitate this reaction. They can decrease reaction time and increase yield compared to benzyl alcohols with electron donating substituents.

After reaction, the bis ionic liquid is easily separated from the reaction medium by washing with distilled water (ionic liquid is soluble in water). The washed bis ionic liquid is distilled under vacuum to recover solvent for reuse in subsequent reactions.

We propose a possible mechanism for the synthesis of 2 -amino- $4 \mathrm{H}$ chromene derivatives. It is assumed that the reaction may proceed initially through full activation by polarization of benzyl alcohols with [BDBDMIm] $\mathrm{Br}$ to form intermediate 5. Next, benzyl alcohols convert to benzaldehydes $\mathbf{6}$ by oxidation with CAN ( $\left(\mathrm{NH}_{4}\right)_{2} \mathrm{Ce}\left(\mathrm{NO}_{3}\right)_{5}$ ). In fact oxidation state of $\mathrm{Ce}(\mathrm{IV})$ changes to oxidation state in $\left(\mathrm{NH}_{4}\right)_{2} \mathrm{Ce}\left(\mathrm{NO}_{3}\right)_{5}(\mathrm{Ce}(\mathrm{III}))$ and nitric acid ${ }^{25,26}$. Then nucleophilic addition of malononitrile $\mathbf{3}$ to intermediate $\mathbf{6}$ and dehydration affords 7. Then Michael addition of 1-naphthol and finally, tautomerization and dehydration, compound $\mathbf{4}$ was produced (Scheme 2).

In the other study to approve of proposed mechanism, we treated 4-nitrobenzyl alcohol (1 mmol), with $4 \mathrm{mmol} \%$ of [BDBDMIm] Br and $0.05 \mathrm{~g}$ of CAN at room temperature in the absence of other reagent, and we succeed to isolate the 4-nitrobenzaldehyde After 60 minutes with 97\% yield. 


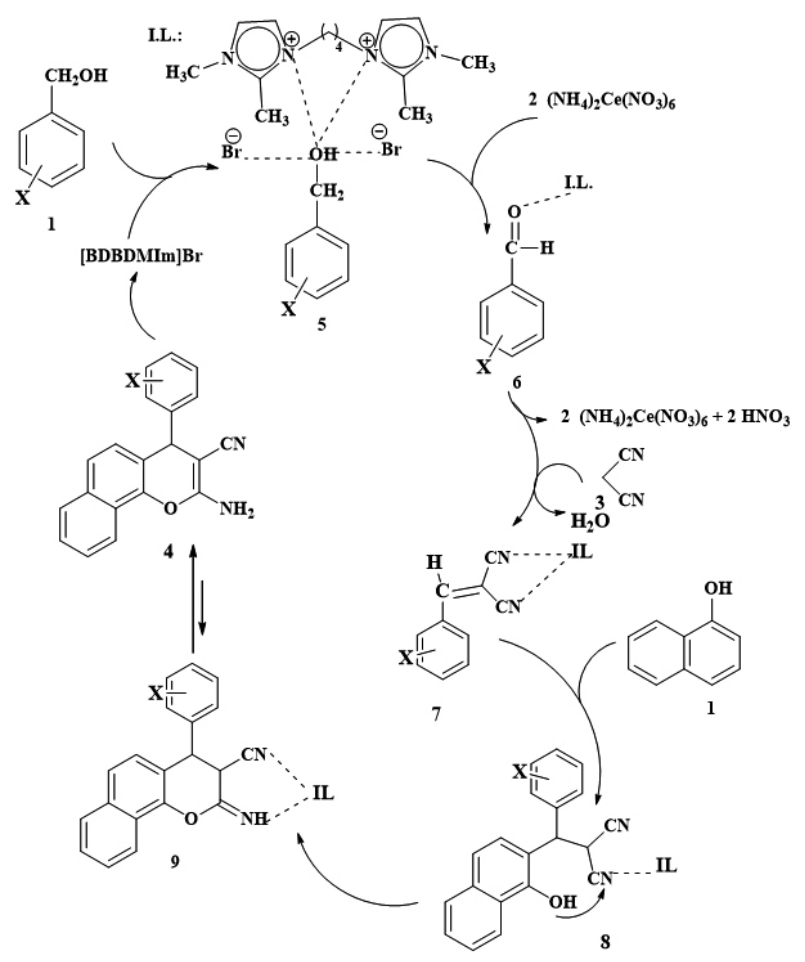

Scheme 2. A possible mechanism for the synthesis of 2-amino- $4 H$ chromenes.

Table 2. Synthesis of 2-amino- $4 H$-chromenes and comparison of efficiency [BDBDMIm]Br.

\begin{tabular}{|c|c|c|c|c|}
\hline Entry & condition & $\mathrm{X}$ & Time (min) & Yield (\%) \\
\hline 1 & $4 a$ & $4-\mathrm{NO}_{2}$ & 60 & 97 \\
\hline 2 & $4 b$ & $4-\mathrm{Cl}$ & 60 & 90 \\
\hline 3 & $4 c$ & $3-\mathrm{OH}$ & 60 & 92 \\
\hline 4 & $4 d$ & 4- $\mathrm{CH}_{3}$ & 75 & 95 \\
\hline 5 & $4 e$ & $3-\mathrm{NO}_{2}$ & 60 & 95 \\
\hline 6 & $4 f$ & $2-\mathrm{NO}_{2}$ & 90 & 88 \\
\hline 7 & $4 \mathrm{~g}$ & $2-\mathrm{Cl}$ & 120 & 82 \\
\hline 8 & $4 h$ & $\mathrm{H}$ & 60 & 90 \\
\hline 9 & $4 i$ & $4-\mathrm{Br}$ & 120 & 86 \\
\hline 10 & $4 j$ & $4-\mathrm{OCH}_{3}$ & 120 & 90 \\
\hline 11 & $4 k$ & $2-\mathrm{OCH}_{3}$ & 120 & 90 \\
\hline 12 & 41 & $3-\mathrm{OCH}_{3}$ & 60 & 92 \\
\hline 13 & $4 \mathrm{~m}$ & $4-\mathrm{OH}$ & 120 & 85 \\
\hline 14 & $4 n$ & $2-\mathrm{OH}$ & 60 & 95 \\
\hline 15 & 40 & $4-\mathrm{I}$ & 60 & 95 \\
\hline 16 & $4 p$ & $3-\mathrm{Br}$ & 60 & 90 \\
\hline
\end{tabular}

${ }^{a}$ All products were characterized by their physical constant, IR, NMR and Elemental analyses. ${ }^{\text {b}}$ Yields based upon starting aldehyde

Our experiments also indicated that after five successive runs, recycled ionic liquid showed no loss of efficiency with regard to reaction time and yield.
Table 3. Evaluation of reusability of ionic liquid for the synthesis of $\mathbf{4 a}$

\begin{tabular}{|c|c|c|c|c|c|}
\hline run & 1 & 2 & 3 & 4 & 5 \\
\hline Time(min) & 60 & 60 & 60 & 60 & 60 \\
\hline Yield(\%) & 97 & 97 & 95 & 97 & 96 \\
\hline
\end{tabular}

Benzaldehyde and other aromatic aldehyde containing electron withdrawing groups (such as nitro, halide) or electron releasing groups (such as hydroxyl, alkoxyl group) were employed and reacted well to give the corresponding 2-amino- $4 H$-chromenes in the yields ranging from 82 to $95 \%$ (Table 1).

\section{ACKNOWLEDGEMENT}

Financial support by Rasht Branch, Islamic Azad University is gratefully acknowledged.

\section{CONCLUSIONS}

Finally, we developed an efficient, green, fast and convenient procedure for the multicomponent synthesis of 2-amino- $4 H$-chromenes through a tandem reaction; First, benzyl alcohols converted to benzaldehydes using a novel oxidant system [BDBDMIm]Br-CAN then, a cyclocondensation reaction of 1-naphthol, aldehydes and malononitrile was carried out. The remarkable advantages offered by this method are that catalyst is inexpensive, non-toxic, easy to handle and reusable. Other most noticeable pluses can be simple workup procedure, short reaction time, high yields of product with better purity and green aspect by avoiding toxic catalyst and hazardous solvent. To the best of our knowledge, this is the first report on synthesis of 2-amino- $4 H$-chromene derivatives using 3,3-(butane-1,4-diyl)bis (1,2-dimethyl-1 $H$-imidazole-3-ium) bromide-CAN.

\section{REFERENCES}

1. Z. Bard, W. Y. Dua, J. Siau, J. Wang, Tetrahedron. Lett. 52, 6137, (2010).

2. L. Moafi. S. Ahadi, A. Bazgir, Tetrahedron. Lett. 51, 6270, (2010).

3. D. J. Maloney, S. M. Hecht, Org. Lett. 7, 4297, (2005).

4. M. Curini, G. Cravotto, F. Epifano, G. Giannone, Curr. Med. Chem. 13, 199, (2006).

5. R. O. Kennedy, R. D. Thornes, Suggested Modes of Action of Coumarins and Some Comments on their Significance. Coumarins: Biology, Applications and Mode of Action, John Wiley \& Sons: Chichester. (1997).

6. G. P. Ellis, In the Chemistry of Heterocyclic Compounds. Chromenes, Chromanes, and Chromones; Weissberger A, Taylor E C Eds, John Wiley: New York, 11, (1977).

7. W. P. Smith, L. S. Sollis, D. P. Howes, C. P. Cherry, D. I. Starkey, N. K. Cobley, J. Med. Chem. 41, 787, (1998).

8. G. A. Kraus, I. Kim, J. Org. Chem. 68, 4517, (2003).

9. K. Hiramoto, A. Nasuhara, K. Michiloshi, T. Kato, K. Kikugawa, Mutat. Res. 395, 47, (1997).

10. J. G. Tangmouo, A. L. Meli, J. Komguem, V. Kuete, F. N. Ngounou, D. Lontsi, V. P. Beng, M. I. Choudhary, B. L. Sondengam, Tetrahedron. Lett. 47, 3067, (2006).

11. R. O. S. Kitamura, P. Romoff, M. C. M. Young, M. J. Kato, J. H. G. Lago, Phytochemistry. 67, 2398, (2006).

12. S. Makarem, A. A. Mohammadi, A. R. Fakhari, Tetrahedron Lett. 49, 7194, (2008).

13. Zh. Zhou, F. Yang, L. Wu, A. Zhan, Chem. Sci. Trans. 1, 57, (2012).

14. S. Khaksar, A. Rouhollahpour, S. Mohammadzadeh Talesh, J. Fluorine. Chem. 141, 11, (2012).

15. S. R. Kale, S. S. Kahandal, A. S. Burange, M. B. Gawande, R. V. Jayaram, Catal. Sci. Technol. 3, 2050, (2013).

16. K. Gong, H. L. Wang, J. Luo, Z. L. Liu, J. Hetero. Chem. 46, 1145, (2009).

17. S. K. Kundu, J. Mondal, A. Bhaumik, Dalton. Trans. 42, 10515, (2013).

18. T. Welton, Chem. Rev. 99, 2071, (1999).

19. R. Sheldon, Chem. Commun. 2399, (2001).

20. J. K. Lee, M. J. Kim, J. Org. Chem. 67, 6845, (2002).

21. M. Nikpassand, L. Zare Fekri, P. Farokhian, Syn. Commun. 45, 2303, (2015).

22. M. Nikpassand, M. Mamaghani, F. Shirini, K. Tabatabaeian, Ultrason. Sonochem. 17, 301, (2010) 
J. Chil. Chem. Soc., 62, No 1 (2017)

23. M. Nikpassand, L. Zare, M. Saberi, Monatsh. Chem. 143, 289, (2012).

24. L. Zare Fekri, M. Nikpassand, K. Hassanpour, Curr. Org. Chem. 12, 76 , (2015).
25. Nair, V. Deepthi, A. Chem. Rev. 107, 1862, (2007).

26. W. S. Trahanovsky, L. B. Young, G. L. Brown, J. Org. Chem. 32, 3865, (1967). 